\title{
Coexisting narcolepsy (with and without cataplexy) and multiple sclerosis
}

Six new cases and a literature review

Ulf Kallweit*, MD;1,2 Claudio L. Bassetti*, MD, Prof.;1 Michael Oberholzer, MD; ${ }^{1}$ Rolf

Fronczek, MD, PhD;3 Mathieu Béguin; ${ }^{1}$ Matthias Strub, MD; ${ }^{4}$ Gert Jan Lammers, MD,

Prof. 3,5

${ }^{*}$ co-shared first authors.

1: University Hospital Bern, Department of Neurology, University Hospital, Inselspital,

Bern, Switzerland

2: University Witten/Herdecke, Institute for Immunology, Witten, Germany

3: Leiden Medical University Center and University of Leiden, Leiden, The Netherlands

4: Practice for neurology, Basel, Switzerland

5: Sleep-wake center SEIN, Heemstede, The Netherlands

Correspondance: Claudio L. A. Bassetti, M.D.

Chairman and Head, Department of Neurology; University Hospital; Inselspital,

Freiburgstrasse 18; 3010 Bern; Switzerland; Telefon: +41 (0)31632 30 66;

E-Mail: claudio.bassetti@insel.ch

ORCID-ID (Dr. Ulf Kallweit): 0000-0003-1975-6919

Word count: Abstract: 201 (max. 250); Text: 2547 words; No of Tables: 3 


\section{Abstract}

Background: There are increasing data suggesting the involvement of the immune system in narcolepsy. The co-occurrence of narcolepsy with other autoimmune disorders (including multiple sclerosis, MS) is rare.

Patients and methods: International multicenter sleep center survey and literature review on narcolepsy with (NC) and without (NwC) cataplexy.

Results: A total of 26 patients (pts), 6 in the survey and 20 in the literature were found. Two different type of association were identified: 1 . Symptomatic type (5 pts): MS preceding the onset of narcolepsy, which was always without cataplexy (NwC); sleep onset REM episodes (SOREM) and hypocretin deficiency were observed in some, and lesions in the hypothalamus in all patients. 2. Coexisting type (18 pts): MS preceding or following the appearance of NC with SOREM, hypocretin deficiency but no lesions in the hypothalamus. A positive effect of steroids, immunoglobulins or natalizumab on narcolepsy symptoms was observed in 4 patients.

Discussion: Narcolepsy and MS are rarely associated. In addition to NwC secondary to hypothalamic demyelination, some patients present a coexistence of MS with NC without detectable hypothalamic lesions. The rarity of reports on this association probably reflects underrecognition. The elucidation of underlying genetic and immune mechanisms needs further studies.

Key words: narcolepsy, cataplexy, multiple sclerosis, autoimmunity, hypocretin, oligoclonal bands, hypothalamus 


\section{Introduction}

Narcolepsy with cataplexy (NC, narcolepsy type 1 (NT1)) is a chronic neurological disorder characterized by wake and sleep dysregulation (excessive daytime sleepiness (EDS), disturbed nocturnal sleep), and motoric (cataplexy, sleep paralysis, REM sleep behavior disorder), neuropsychiatric (hallucinations, depression) and metabolic/ endocrinological (obesity, precocious puberty) symptoms. In some patients narcolepsy present without cataplexy (NwC); often cataplexy develops after a delay of years.

Narcolepsy (NC and NwC) approximately affects $0.026-0.05 \%$ of the population in Europe and Northern America. Prevalence is found to be higher in Japan $(0.59 \%)$ and lower in Israel (0.00023\%). [1] Incidence rate is about $0.6-1 / 100.000$ person-years.

Based upon genetic, epidemiological and immunologic studies, narcolepsy is suggested to be an immune-mediated disorder. [2] NC is caused by the selective loss of hypocretin cells of the posterior hypothalamus that produce the neuropeptide hypocretin (HCRT). Correspondingly, cerebrospinal fluid (CSF) hypocretin-1 (hcrt-1) levels are low/undetectable in $\square 90 \%$ of NC patients and in $20-30 \%$ of patients with NwC. [3] Patients today diagnosed as having narcolepsy with cataplexy probably have in some Commented [GJ1]: without cases (those with HLA positivity and hypocretin deficiency) a similar etiopathophysiology as narcolepsy with cataplexy, in a significant number of cases its origine is however unclear.

Immune dysfunction for narcolepsy with cataplexy is suspected on the basis of a strong association (>98\% for NC) with human leukocyte antigen (HLA) DQB1*06:02, T-cell receptor (TCR) and P2RY11 polymorphisms, increased levels of Trib2-specific, and anti-streptolysin $\mathrm{O}$ antibody titers, and alterations in the cytokine profile. [2] Epidemiological and immunological observations indicate that different infections 
(Streptococcus, H1N1) and vaccination with Pandemrix ${ }^{\mathrm{TM}}$ are potential triggering factors.

Narcolepsy-like phenotypes are also described as paraneoplastic syndrome. In one of these cases, a CD8+ inflammatory-mediated response against hypocretin neurons was found. Special focus has been put on T-cell immune pathology in narcolepsy. Destruction of hypocretin producing neurons may be mediated by autoantigen specific CD4(+) T-cells or superantigen stimulated CD8(+) T-cells, or independent of T-cells by activation of DQB1*0602 signaling. [2,4]

MS is a chronic inflammatory disease of the central nervous system (CNS) characterized by inflammation, demyelination, axonal injury and axonal loss. It is believed to be an autoimmune disorder, but the antigen specificity of the immune response is unknown. Autoreactive T cells activated outside the CNS cross the bloodbrain-barrier and are reactivated by local antigen-presenting cells. [4] Genetic susceptibility to MS is associated with the HLA region located on the short arm of chromosome 6 (6p21). Variations in this region can be positively or negatively associated with disease risk and disease course. The HLA class II region has the largest influence, with HLA-DRB1*1501 being the single strongest susceptibility locus, conferring a threefold increase in the MS risk. The frequency of the human leukocyte antigen $\mathrm{DQB} 1{ }^{*} 0602$ was consistently found to be more prevalent (50-60\% vs. $\left.20-30 \%\right)$ in MS cases than in controls. [5,6] In MS, fatigue (>80\%) is common, and EDS and sleep fragmentation are found in about $12-30 \%$ and $30-74 \%$, respectively. [7]

\section{Aims}

The occurrence of narcolepsy and autoimmune disorders (including MS) is a controversial issue. Anecdotally reports described the coexistence of narcolepsy with autoimmune disorders including multiple sclerosis. In a first systematic study, however, 
no increased frequency of autoimmune disorders was found in patients with narcolepsy (with and without cataplexy) [8] and only one case was reported to have both narcolepsy and MS.

We aimed at investigating frequency, characteristics and potential interactions of coexisting narcolepsy and MS a) in the literature and b) in a multi-center, international database survey.

\section{Patients and methods}

Retrospective sleep database multicenter survey in three large tertiary sleep centers with longstanding expertise in narcolepsy (Bern $(\mathrm{CH})$, Leiden (NL), Witten/Hagen (DE)) for cases of coexisting narcolepsy and MS. This survey includes a population of approx. 1200 narcolepsy patients. For diagnosis of narcolepsy, ICSD-3 criteria have been applied. The databases contain all patients diagnosed in the respective centers (during more than 15 years in Leiden and Bern, and during 8 years in Witten) and are regularly updated, also on comorbid disorders such as MS.

For epidemiological data, a PubMed research was conducted for topical literature published until 30 June 2017. Search terms included narcolepsy, narcolepsy with and w/o cataplexy, cataplexy, NT1, NT2, hypocretin, orexin, multiple sleep latency test, Multiple Sclerosis, MS, encephalomyelitis disseminata, and disseminated sclerosis.

In addition, review of published articles on comorbidities and/or coexisting autoimmune disorders of narcolepsy were used as primary resources.

For grading and comparison, an estimation of co-existing cases of narcolepsy and MS has been calculated based upon available epidemiological data. 
Ethics: The manuscript only includes data from a retrospective database analysis and literature search. Local ethical committees have approved the use of the database.

\section{Results}

\section{Patients from survey of 3 narcolepsy centers}

We identified six patients with both narcolepsy and multiple sclerosis. All had cataplexy and were coexisting cases.

For details please see Table 1.

\section{$\underline{\text { Patients from literature review }}$}

Our research revealed 145 articles. After evaluation for content and relevance, 20 patients (of whom 11 with cataplexy) could be identified. For details please see Table 2.

\section{Epidemiology:}

For comparability, prevalence data from Europe were selected. According United Nations (UN, 2015), Europe has 738 millions of habitants. European prevalence rates for narcolepsy (with cataplexy) and Multiple Sclerosis (all types) were used for calculation of overlapping cases (without projecting a potential causal or mechanistic association).

For Europe calculated number of overlapping cases is between 77 and 1513 with a middle $50 \%$ range between 475 and 1115 people.

For details see Table 3.

Taken these numbers, the projected estimated number of cases is between 500 and 8000 worldwide.

\section{$\underline{\text { Classification }}$}


After evaluation of all patients (new cases $(n=6)$ and published cases $(n=20)$ ), we identified two different types of association between narcolepsy and multiple sclerosis. Articles in Table 2 are marked for types. Three out of 20 literature cases could not be classified due to lack of information.

\section{Symptomatic narcolepsy without cataplexy secondary to hypothalamic demyelination}

The two key features of this group are the presence of EDS as exclusive symptom of narcolepsy $(n=5 / 5)$ and the occurrence of uni- or bilateral MS lesions in the hypothalamus $(n=5 / 5)$. In no patient, cataplexy was reported whereas hypocretin levels were low/undetectable in 3 out of 5 pts. HLA-DQB1*0602 was positive in 0/2 pts. Sleepwake tests were available only in 2 patients: the first one had "SOREM periods" (no additional MSLT information available) and the second one had a normal mean sleep latency in MSLT and no SOREM periods. Oligoclonal bands, a typical finding in MS, were found in $1 / 1$ patient. Narcolepsy appeared after the onset of MS $(n=3 / 5$, no data available in 2 patients). In one patient, steroid treatment (5 days, $500 \mathrm{mg} / \mathrm{d}$, intravenously) improved EDS for more than 18 months.

\section{Coexisting narcolepsy with cataplexy and relapsing remitting MS but without}

\section{hypothalamic demyelination}

The key features of this group are the presence of narcolepsy with cataplexy and relapsing remitting (RR) MS in the absence of hypothalamic MS lesions. EDS was present in all patients $(n=18)$, cataplexy in 17 of them. Sleep-wake tests were characteristic for narcolepsy in all patients tested $(n=16 / 16)$. Low/undetectable CSF hypocretin levels were found in 6/6 patients. HLA haplotype status for DQB1*0602 (or DR2) was positive in 9 out of 9 patients and in 4/4 for HLA-DRB1*1501. In all patients clinical symptoms and course were typical for RRMS. MRI findings included the 
absence of lesions in the hypothalamus $(n=14 / 14)$ but the presence of different supraand (rarely) infratentorial and spinal cord lesions. Oligoclonal bands,a typical finding in MS, were found in most patients $(n=8 / 9)$. In $9 / 18$ pts MS preceded, in 9 patients followed the onset of narcolepsy.

Five patients were treated with disease modifying MS treatments: natalizumab $(n=2)$, beta-interferons $(n=2)$ and glatirameracetate $(n=1)$. In one patient, ivig treatment, in one patient natalizumab therapy and in one patient, long-term steroid therapy resulted in an improvement of EDS.

\section{Discussion}

Our series of coexisting narcolepsy and multiple sclerosis is the largest series published so far. We identified two groups of patients. Secondary cases typically present with EDS and MS lesions in the hypothalamus but no cataplexy (NwC). Conversely, coexisting cases present with both EDS and cataplexy in the absence of hypothalamic lesions (but otherwise typical findings of RRMS).

The reported cases with coexisting narcolepsy and MS are scarce $(n=20$, since 1926). This contrasts with the results of our survey, showing a number within the calculated range for coexistence based on the prevalence of both disorders, This discrepancy with the cases published in the literature may be due to different reasons. Narcolepsy is an underdiagnosed disorder in general. In addition, narcolepsy in MS is probably also underdiagnosed because fatigue, tiredness, muscle weakness and disturbed nocturnal sleep are frequent in MS.

Although epidemiologic considerations alone do not allow pathophysiologic considerations we suggest that, based upon additional indirect evidence (see below), a 
The observation of secondary narcolepsy in the context of hypothalamic damage is not surprising. [9] It is of interest, however, that only rarely, demyelinating disease involving the hypothalamus was reported to give rise to narcolepsy, typically without cataplexy. In fact, we did not find as single case of narcolepsy with cataplexy with a defined hypothalamic lesion due to MS. Noteworthy, NC was described in few patients with neuromyelitis optica (NMO) and involvement of the hypothalamus. [10]

The possibility of a co-occurrence of both disorders through possibly shared underlying immunological mechanisms should be discussed. This could result from genetic factors (e.g. HLA DQB1*0602 and DRB1*1501), the environment or an interaction of the two. Ekbom reported the exceptional association of familial MS and NC in a single family. [11] The rarity of this association despite the presence of similar HLA predisposition may be due to differences in (other) predisposing and protective genes. In study of 18 MS patients no association was found between HLA DQB1*0602 positivity (found in 6 of them) and narcolepsy like features. However, two out of 6 HLA patients also carried a protective gene (DQB1*02). [12] The need of other factors besides DRB1*15:01/DQB1*06:02 to develop either one of the two disorders is underlined by the report of monozygotic twins, one of which developed narcolepsy with cataplexy whereas the other one presented with the other multiple sclerosis. [13] In narcolepsy HLA-DRB1*03- DQB1*02 and HLA-DQB1*0603 were reported to be protective. [14] However, in MS HLA-DRB1*03:01 is one of the risk alleles [15] and HLA DQB1*0602 as well as DQB1*0603 are associated with more severe damage on inflammatory and neurodegenerative MRI measures in MS. [16] Hence, risk genes for MS (or narcolepsy) might have a protective function for narcolepsy (or MS) either by being protective themselves or by allele competition. [17] In fact, other protective mechanisms besides the genetically determined HLA predisposition are probably necessary and include specific immune pathways and reactions (possibly $\mathrm{T}$ cell mediated) that arise in MS and 
influence the vulnerability to develop narcolepsy with cataplexy (or vice versa). In a study profiling the autoantibody repertoire in plasma/serum MS and NC samples using high-density peptide microarrays, different reactivity patterns between and among the narcolepsy and MS-related sample groups were found. [18] Myelin oligodendrocyte glycoprotein (MOG) has been suggested to have a central role in the regulation of tolerance and autoimmunity. MOG antibodies have been described in patients presenting with acute demyelinating disease of the central nervous system, often previously labeled under MS syndrome. [19] Interestingly, a MOG missense mutation has been identified as cause of familial narcolepsy with cataplexy. [20] In a recent study (Latorre D et al., submitted), we could provide additional evidence for the autoimmune pathogenesis of narcolepsy. We used combined antigenic stimulation, $\mathrm{T}$ cell cloning, and TCR sequencing in order to interrogate memory $\mathrm{T}$ cells from narcolepsy patients and found autoreactive CD4+ T cells specific for HCRT in 18/19 patients, but only rarely in healthy donors. Memory CD8+ T cells specific for HCRT and memory CD4+ T cells specific for TRIB2, a self-antigen expressed by hypocretin-producing neurons, were also found in some patients. Highest frequency of HCRT-reactive T cells were found in a patient with coexisting NC and MS. Our results establish that CD4+ / CD8+ T cell reactivity to self-antigens expressed by HCRT-producing neurons is associated with narcolepsy. [21]

The impact of intravenous immunoglobulin (ivig) and natalizumab therapy on EDS in two of our patients is interesting and may point to some effect of immunomodulatory treatments in single/selected patients with narcolepsy. In line with this, an improvement of fatigue and also EDS in MS patients by natalizumab treatment was recently shown. [22, 23]

Our study points to the possibility of a direct/causative link between narcolepsy and MS and by this further supports the hypothesis of an autoimmune pathophysiology 
of narcolepsy. More research is needed to elucidate the nature of this link (including the possible existence of mechanisms predisposing and/or protecting for both diseases) and the potential implications for future (immunomodulatory?) treatments of narcolepsy.

\section{Conflict of interest:}

The authors declare that they have no conflict of interest.

\section{References:}

1. Ohayon MM, Priest RG, Zulley J, Smirne S, Paiva T. Prevalence of narcolepsy symptomatology and diagnosis in the European general population. Neurology 2002;58:1826-33.

2. Liblau RS, Vassalli A, Seifi A, Tafti M. Hypocretin ( orexin ) biology and the pathophysiology of narcolepsy with cataplexy. Lancet Neurol 2015;14:318-28.

3. Andlauer $\mathrm{O}$, Moore $\mathrm{H}$, 4th, Hong SC, et al. Predictors of hypocretin (orexin) deficiency in narcolepsy without cataplexy. Sleep. 2012;35:1247-55.

4. Bernard-Valnet R, Yshii L, Quériault C, et al. CD8 T cell-mediated killing of orexinergic neurons induces a narcolepsy-like phenotype in mice. Proc Natl Acad Sci U S A. 2016;113:10956-61.

5. Kamm CP, Uitdehaag BM, Polman $\mathrm{CH}$. Multiple sclerosis: current knowledge and future outlook. Eur Neurol. 2014;72:132-41.

6. Schmidt H, Williamson D, Ashley-Koch A. HLA-DR15 haplotype and multiple sclerosis: a HuGE review. Am J Epidemiol. 2007;165:1097-109.

7. Brass SD, Li CS, Auerbach S. The underdiagnosis of sleep disorders in patients with multiple sclerosis. J Clin Sleep Med. 2014;10:1025-31. 
8. Barateau L, Lopez R, Arnulf I, et al. Comorbidity between central disorders of hypersomnolence and immune-based disorders. Neurology 2017;88:93-100.

9. Nishino S, Kanbayashi T. Symptomatic narcolepsy, cataplexy and hypersomnia, and their implications in the hypothalamic hypocretin/orexin system. Sleep Med Rev. 2005;9:269-310.

10. Silber MH. Autoimmune sleep disorders. Handb Clin Neurol. 2016;133:317-26.

11. Ekbom K. Familial multiple sclerosis associated with narcolepsy. Arch Neurol. $1966 ; 15: 337-44$

12. Lorenzoni PJ, Werneck LC, Crippa AC,et al. Is there a relationship between narcolepsy, multiple sclerosis and HLA-DQB1*06:02? Arq Neuropsiquiatr. 2017;75:345-348.

13. Jennum PJ, Kornum BR, Issa NM,et al. Monozygotic twins discordant for narcolepsy type 1 and multiple sclerosis. Neurol Neuroimmunol Neuroinflamm. 2016;3:e249.

14. Hor H, Kutalik Z, Dauvilliers Y, et al. Genome-wide association study identifies new HLA class II haplotypes strongly protective against narcolepsy. Nat Genet. 2010;42:786-9.

15. Moutsianas L, Jostins L, Beecham AH, et al. Class II HLA interactions modulate genetic risk for multiple sclerosis. Nat Genet. 2015; 47:1107-13.

16.Zivadinov R, Uxa L, Bratina A, et al. HLA-DRB1*1501,-DQB1*0301,-DQB1*0302, -DQB1*0602, and -DQB1*0603 alleles are associated with more severe disease outcome on MRI in patients with Multiple Sclerosis. Int Rev Neurobiol 2007;79:521- 35

17. Tafti M, Hor H, Dauvilliers Y, Lammers GJ, Overeem S, Mayer G. DQB1 locus alone explains most of the risk and protection in narcolepsy with cataplexy in Europe. Sleep 2014;37:19-25. 
18. Zandian A, Forsström B, Häggmark-Månberg A, et al. Whole-Proteome Peptide Microarrays for Profiling Autoantibody Repertoires within Multiple Sclerosis and Narcolepsy. J Proteome Res. 2017;16:1300-1314

19.Zabad RK, Stewart R, Healey KM. Pattern Recognition of the Multiple Sclerosis Syndrome. Brain Sci. 2017;7. pii: E138.

20. Hor H, Bartesaghi L, Kutalik Z, et al. A missense mutation in myelin oligodendrocyte glycoprotein as a cause of familial narcolepsy with cataplexy. Am J Hum Genet. 2011;89:474-9.

21. Latorre D, Kallweit U, Armentani E, et al. Autoreactive T cells in narcolepsy patients target antigens of hypocretin-producing neurons. Submitted.

22. Penner I-K, Sivertsdotter EC, Celius EG, et al. Improvement in Fatigue during Natalizumab Treatment is Linked to Improvement in Depression and Day-Time Sleepiness. Front Neurol. 2015;6:18.

23. Sater RA, Gudesblatt M, Kresa-Reahl K, Brandes DW, Sater P. NAPS-MS: Natalizumab Effects on Parameters of Sleep in Patients with Multiple Sclerosis. Int J MS Care. 2016;18:177-82. 